Meta

Journal des traducteurs

Translators' Journal

\title{
Quelques repères épistémologiques pour une approche cognitive de la traduction. Application à la traduction spécialisée en biomédecine
}

\section{Sylvie Vandaele}

Volume 52, numéro 1, mars 2007

Traductologie : une science cognitive

URI : https://id.erudit.org/iderudit/014728ar

DOI : https://doi.org/10.7202/014728ar

Aller au sommaire du numéro

Éditeur(s)

Les Presses de l'Université de Montréal

ISSN

0026-0452 (imprimé)

1492-1421 (numérique)

Découvrir la revue

Citer cet article

Vandaele, S. (2007). Quelques repères épistémologiques pour une approche cognitive de la traduction. Application à la traduction spécialisée en biomédecine. Meta, 52(1), 129-145. https://doi.org/10.7202/014728ar
Résumé de l'article

Le présent article fait état de chercheurs et de courants de pensée clés qui pavent le chemin de nouvelles recherches en traductologie dans une perspective cognitive : Spinoza au plan philosophique, Damasio et Edelman en neurosciences, la Gestalt et les travaux de Lakoff, de Talmy et de Fauconnier en sémantique cognitive. Nous en situerons les principaux concepts au sein des sciences cognitives et nous expliquerons pourquoi ils nous paraissent essentiels pour la pratique de la traduction et la traductologie. Quelques résultats tirés de nos travaux sur les modes de conceptualisation en biomédecine illustreront l'approche proposée. 


\title{
Quelques repères épistémologiques pour une approche cognitive de la traduction. Application à la traduction spécialisée en biomédecine*:
}

\author{
SYLVIE VANDAELE \\ Université de Montréal, Montréal, Canada \\ sylvie.vandaele@umontreal.ca
}

\begin{abstract}
RÉSUMÉ
Le présent article fait état de chercheurs et de courants de pensée clés qui pavent le chemin de nouvelles recherches en traductologie dans une perspective cognitive: Spinoza au plan philosophique, Damasio et Edelman en neurosciences, la Gestalt et les travaux de Lakoff, de Talmy et de Fauconnier en sémantique cognitive. Nous en situerons les principaux concepts au sein des sciences cognitives et nous expliquerons pourquoi ils nous paraissent essentiels pour la pratique de la traduction et la traductologie. Quelques résultats tirés de nos travaux sur les modes de conceptualisation en biomédecine illustreront l'approche proposée.
\end{abstract}

\begin{abstract}
This paper presents some key researchers and theories that open the way for new types of research in translatology using a cognitive approach: Spinoza in philosophy, Damasio and Edelman in neurosciences, the Gestalt and the work of Lakoff, Talmy and Fauconnier in cognitive semantics. The main concepts will be placed within the cognitive science framework and we will explain why they are important for the practice of translation as well as in research. A few results taken from our work on conceptualisation modes in biomedicine will illustrate the proposed approach.
\end{abstract}

MOTS-CLÉS/KEYWORDS

traductologie cognitive, philosophie, neurosciences, sémantique cognitive, modes de conceptualisation

Ce monde regorge d'intentionnalité, de projections, de sentiments, de préjugés et d'affection.

G. M. Edelman, Biologie de la conscience, p. 378 (Brilliant Air, Brilliant Fire: On the Matter of Mind, traduction de A. Gershenfeld, 1992).

\section{Introduction}

La progression des sciences cognitives au cours des vingt dernières années a permis de nourrir une réflexion multidisciplinaire aux frontières des neurosciences, de la psychologie, de la philosophie, de la linguistique et de l'intelligence artificielle. La traductologie, quant à elle, n'a pas seulement à s'inspirer des travaux menés dans la mouvance cognitive, elle est dans une situation idéale pour y contribuer à part entière. Traduire ou interpréter, c'est mobiliser certains des aspects les plus divers, les plus profonds et les plus complexes de la pensée humaine. La pratique et l'enseignement 
de la traduction, que ce soit en littérature ou dans les domaines spécialisés, peuvent ainsi être envisagés sous cet angle, et venir ainsi enrichir les autres courants qui ont alimenté l'affermissement et l'autonomisation de la discipline.

Un repérage épistémologique s'impose cependant. Le domaine du «cognitif» couvre en effet un vaste ensemble de tendances complexes pouvant s'opposer radicalement. Dans le présent article, nous chercherons - modestement au regard de l'ampleur des domaines concernés - à proposer quelques repères permettant d'orienter la réflexion dans une voie qui nous semble être des plus fructueuses. Bien que nous les suivions dans le cadre de nos travaux sur la traduction et la terminologie en biomédecine, ils nous paraissent de portée générale et sont donc susceptibles de s'appliquer aux différents contextes de traduction. Dans le présent article, la filiation philosophique à laquelle se rattache notre réflexion sera tout d'abord présentée, puis les courants des neurosciences et de linguistique qui en découlent seront distingués. Nous illustrerons le choix des cadres théoriques proposés à l'aide de quelques exemples tirés de nos travaux et nous conclurons par quelques remises en question et une mise en perspective dans le cadre de la traductologie.

\section{De Spinoza aux neurosciences du XXI ${ }^{\mathrm{e}}$ siècle}

\subsection{Spinoza contre Descartes}

$\mathrm{Au} \mathrm{XVII}^{\mathrm{e}}$ siècle, Spinoza s'oppose à Descartes, de 36 ans son aîné, et à sa doctrine dualiste. Tous deux sont exilés à Amsterdam, essentiellement pour des raisons politiques et religieuses. Dans le Discours de la méthode, Descartes (1637) postule un corpsmachine, c'est-à-dire un automate animé par des réflexes. L'âme, définie comme une substance pensante indépendante du corps, est donnée par Dieu à l'homme pour le distinguer de l'animal. Elle est le principe créateur de la pensée et du langage. Ainsi se fonde la pensée dualiste occidentale autorisant, notamment, l'objectivisation de l'objet scientifique indépendante du sujet, l'exclusion de la participation du corps à la pensée, et l'autonomie supposée du fonctionnement de l'esprit.

Spinoza adopte une optique radicalement opposée, son souci premier étant le rapport entre l'homme et la nature. Celle-ci a un caractère «substantiel», elle est cause d'elle-même et non œuvre d'un Dieu qui lui est distinct. De la vaste pensée de Spinoza, qui couvre l'exégèse religieuse, la politique et la philosophie, nous retiendrons ici l'affirmation de l'unicité du corps et de l'esprit, dans laquelle Damasio, un neurologue, voit l'annonce de la biologie moderne:

En refusant de fonder l'esprit et le corps sur des substances différentes, du moins Spinoza exprimait-il son opposition à la vision du problème de l'esprit et du corps qui prévalait à son époque. Son désaccord tranchait sur une mer de conformisme. Plus étonnante était son idée selon laquelle l'esprit et le corps sont une seule et même chose. Cela ouvre une saisissante possibilité. Il se pourrait ainsi que Spinoza ait eu l'intuition des principes régissant les mécanismes naturels responsables des expressions parallèles de l'esprit et du corps. [...] je suis convaincu que les processus mentaux ont leur fondement dans les encartages cérébraux du corps, c'est-à-dire dans des structures neurales représentant les réponses aux évènements qui causent les émotions et les sentiments. (Damasio 2003: 19)

Son Traité théologico-politique ayant suscité de violentes controverses, Spinoza ne publie plus. Ce n'est qu'après sa mort que ses œuvres sont éditées par ses amis, circulent 
anonymement, et inspirent - plus ou moins secrètement, semble-t-il - les penseurs des Lumières (peut-être même plus que Locke). Elles menacent de disparaître sous l'effet des attaques incessantes, mais Spinoza suscite l'enthousiasme de Goethe, de Wundt et de von Hemholtz, inspire Einstein, peut-être même Freud (Damasio 2003: 260-266). Il est maintenant redécouvert par différents chercheurs de la neurobiologie et des sciences cognitives, de la philosophie et de la psychologie (Changeux et Ricœur 1998; Atlan 2002; Rizk 2006; Jaquet 2004; Vendewalle et coll. 2003).

\subsection{L'unité du corps et de l'esprit: quelles neurosciences?}

$\mathrm{Au}$ sein des sciences cognitives et des neurosciences en particulier, l'opposition entre les courants se rattachant aux pensées cartésienne et spinoziste est très marquée et se situe au cour d'un débat complexe qui n'est pas terminé (voir par ex. Le Ny 2005: 24-26; Fuchs 2004). L'enjeu fondamental est la compréhension du fonctionnement de l'esprit et du cerveau et la façon de concevoir l'émergence de la conscience, avec de nombreuses répercussions dans tous les domaines, médecine, intelligence artificielle ou éducation, voire la vision de la société dans son ensemble.

Le courant dualiste cartésien, cohérent avec l'idéalisme platonicien, mène au cognitivisme dit classique, ou symbolique, issue de la cybernétique, avec Turing, Putnam, Fodor et Pylyshin: c'est la théorie computationnelle de l'esprit, selon laquelle le fonctionnement cognitif serait semblable à celui d'un ordinateur fonctionnant selon des algorithmes manipulant logiquement des structures syntaxiques. Pour ce faire, l'esprit devrait être organisé en modules (Fodor 1983). Même si Fodor a luimême nuancé ce concept (Fodor 2000), l'idée selon laquelle le cerveau n'est ni plus ni moins qu'un ordinateur manipulant syntaxiquement des symboles reste chez lui une idée forte, de même que chez de nombreux chercheurs en sciences cognitives. Permettant de décomposer les activités cognitives en composantes plus ou moins indépendantes traitées séquentiellement, le modèle modulaire a donné lieu à des versions plus ou moins fortes et a, de fait, permis de nombreuses études tant en psychologie qu'en neurosciences (voir Rouveret 2004).

Le courant connexionniste, issu lui aussi de la cybernétique, est d'inspiration empirique et se rapproche davantage de la neurobiologie, en ce qu'il se raccroche à l'idée de connexions neuronales multiples fonctionnant de façon parallèle et distribuée. Il s'est proposé, dans les années 1980, comme solution de rechange au courant symbolique, mais il reste fortement inspiré par l'intelligence artificielle (Varela 1989).

Se fondant sur une connaissance approfondie de la biologie neuronale, Edelman (neurobiologiste prix Nobel de médecine) s'oppose vigoureusement au modèle computationnel symbolique, tout comme aux modèles connexionnistes (Edelman 1992: 349): "Il s'agit là de modèles formels dans lesquels les connexions entre éléments du réseau subissent des modifications qui sont grosso modo analogues à celles qui surviennent dans les synapses. [...] cette métaphore devient exagérée [...]», essentiellement parce que «les modèles connexionnistes exigent qu'un programmeur ou un opérateur spécifient leurs entrées et leurs sorties, et ils font appel à des algorithmes pour l'introduction de ces spécifications». Pour Edelman, cette caractéristique est rédhibitoire: de telles architectures ne correspondent pas à la réalité biologique. Bien que l'idée de l'émergence de la conscience à partir de la matière vivante semble proche du concept constructiviste d'énaction proposé par Varela (1989), ultimement, le 
connexionnisme se rapproche des modèles computationnels de Turing. En fait, le cerveau serait plutôt un système sélectif mettant en jeu des "cartes» de neurones se construisant et se renforçant au cours du développement:

Il en résulte une entité [le cerveau] subtile et multistratifiée, pleine de boucles et de niveaux différents. Des gènes aux protéines, des cellules aux développements orchestrés, de l'activité électrique à la libération de neuromédiateurs, des couches sensorielles aux cartes, de la forme à la fonction et au comportement, et en sens inverse, de la communication sociale vers n'importe lequel de ces niveaux ou vers tous à la fois, nous nous trouvons devant un système de sélection somatique constamment soumis à la sélection naturelle $[\ldots]$. Dans ces conditions, il n'est pas étonnant que les philosophes, qui réfléchissent au problème de l'esprit sans disposer de ces connaissances, aient été tentés de postuler l'existence de certaines entités, que les physiciens soient tentés de postuler de nouveaux champs matériels exotiques, ou que ceux qui aspirent à l'immortalité continuent à postuler l'existence d'esprits éternels. (Edelman 1992: 228)

Dans cette perspective, le cerveau est un "corrélateur», c'est-à-dire qu'il établit des «corrélations entre les entrées temporelles au cours de son propre développement et, au cours de son fonctionnement à l'âge adulte, des corrélations entre les propriétés des signaux et des scènes pour donner naissance à la conscience» (Edelman 1992: 230).

Cette position accorde la prééminence à l'organisation biologique de laquelle émergent la conscience et l'intelligence. Est-elle la marque de «matérialistes sophistiqués », comme se définit lui-même Edelman (1992: 247) ? On pourrait aussi l'envisager, à la façon de Spinoza, comme panthéisme. Nous ne prendrons pas position sur ce point, car ce jeu se situe au plan des convictions personnelles hors d'atteinte de la réflexion scientifique. Ce qui importe est la primauté du réel, en opposition avec le constructivisme extrême proche de l'idéalisme ${ }^{1}$. Edelman considère en effet comme «[... hypothèses de base de toute vision scientifique:

1. Il existe un monde réel - un monde décrit par les lois de la physique, qui s'appliquent partout. (C'est l'hypothèse physique.)

2. Nous sommes partie intégrante de ce monde, nous suivons ses lois et nous sommes le produit d'une évolution à partir d'une origine ancienne. C'est l'apparition, au cours de l'évolution, de nouvelles morphologies qui a donné naissance à l'esprit. (C'est l'hypothèse évolutionniste.)

3. Il est possible de replacer l'esprit dans la nature. Il est possible de construire une science de l'esprit sur des bases biologiques. Et la façon d'éviter les cercles vicieux et les impasses consiste à construire une théorie du cerveau fondée sur des principes sélectionnistes.» (Edelman 1992: 244)

Comment la conscience émerge-t-elle à partir de la matière organique? Un mystère fondamental, vertigineux, persiste malgré tout. Le point fondamental est que l'esprit est contraint par l'organisation biologique, et qu'il n'est pas une entité autonome et désincarnée. L'être vient avant la conscience, c'est je suis donc je pense, plutôt que je pense donc je suis.

La position d'Edelman est compatible avec la philosophie de Spinoza, bien qu'à notre connaissance il ne l'évoque pas. Damasio se réclame de cette filiation, citant à l'appui de son rejet du dualisme cartésien de nombreuses observations cliniques l'amenant à considérer l'intégration du sentiment (feeling) et des émotions ${ }^{2}$ au sein même du processus rationnel. Damasio reformule en quelque sorte la pensée de Spinoza en proposant que «les processus mentaux ont leur fondement dans les encar- 
tages cérébraux du corps» (Damasio 2003: 19). Dans cette perspective, l'intégration corps-esprit permet d'attribuer une valeur cognitive au sentiment et aux émotions, en ce qu'ils permettent de «qualifier» le réel indispensable à la prise de décision rationnelle.

\section{Des neurosciences à la sémantique cognitive}

Différentes linguistiques découlant des courants cognitivistes s'opposent dans leurs fondements et leur pratique (voir par ex. Anscombre1998; Fuchs 2004). Le programme du cognitivisme classique computationnel symbolique a donné lieu aux grammaires génératives chomskyennes (que Lakoff qualifie de "première génération») accompagnées (1) d'une démarche hypothético-déductive faisant appel à des exemples construits grâce à la compétence du linguiste, (2) d'une conception du langage mettant en jeu une correspondance formes linguistiques et symboles et (3) d'une modélisation logico-algébrique fondée sur l'idée de modules (voir Fuchs 2004; Rouveret 2004).

Edelman indique que les théories linguistiques correspondant le mieux à ses positions en matière de neurobiologie sont les grammaires cognitives et plus particulièrement la sémantique expérientialiste de Lakoff (Edelman 1992: 379). De fait, fonder l'étude du langage et des langues sur le concept d'incarnation de l'esprit, appuyé par les neurosciences, fait la part belle à la sémantique. Les grammaires cognitives de «deuxième génération» sont en opposition avec le programme classique. Les représentants de ce courant adoptent une démarche plutôt inductive, reconnaissent l'usage de corpus, et ont recours à différents modèles de type géométrique ou dynamique rénovant la prise en compte de la polysémie et de phénomènes tels que la métaphore, désormais placée au plan de la pensée (voir Fuchs 2004; Victorri 2004). Lakoff plaide en faveur de l'intégration corps-cerveau-esprit et de la prise en compte, au plan linguistique, de la mémoire, de la perception, de l'activité kinesthésique, des interactions sociales et de la culture, de la connaissance du contexte et des besoins communicatifs (Lakoff et Johnson 1999: 473-475). Dans le courant des grammaires cognitives, se démarquent également les travaux de Langacker (que nous n’aborderons pas ici), de Talmy et de Fauconnier.

\subsection{Lakoff: l'expérientialisme}

Lakoff s'inspire de la Gestalt, école de psychologie allemande de la fin du XIX ${ }^{e}$ siècle, fondée en autres par E. Mach (Lakoff 1977). Le verbe gestalten veut dire «mettre en forme», «donner une structure signifiante». Le résultat, la gestalt, est une forme structurée, complète et prenant du sens. Pour illustrer ce principe, prenons l'exemple suivant: supposons que l'on regarde une image constituée par une surface noire parsemée de petits points blancs brillants. On pourrait se demander si l'image représente un ciel étoilé, mais un biologiste cellulaire pourrait y voir aussi une culture de cellules colorées ou marquées par des composés fluorescents. Dès lors que l'on détermine, parmi les points blancs disséminés dans le noir, la forme correspondant à la Grande Ourse (soit par une opération mentale reliant les points ad hoc, soit en dessinant un trait), aucun doute n'est plus possible: il s'agit du ciel étoilé. C'est en reconnaissant une forme dans une surface noire parsemée de points blancs en elle-même dépourvue de sens, que précisément le sens a émergé. Selon ce courant de pensée, notre système 
de perception, de type gestalt, génère des «formes» mentales fondamentales représentant des catégories de base (mouvement, haut/bas, faim, douleur, reconnaissance de ce qui est l'eau, la pierre...), que nous utilisons pour ensuite concevoir le monde à des niveaux de plus en plus abstraits.

Le cognitivisme expérientialiste s'oppose au cognitivisme objectiviste sur différents points fondamentaux. Premièrement, tout comme Edelman, Lakoff postule l'incarnation de la pensée, en ce qu'elle implique l'expérience sensori-motrice:

«Experiential» is to be taken in the broad sense, including basic sensory-motor, emotional, social, and other experiences of a sort available to all normal beings - and especially including innate capacities that shape such experience and make it possible. The term "experience" does not primarily refer to incidental experiences of a sort that individuals happen to have had by virtue of their unique histories. We are focusing rather on that aspect of experience that we have simply by virtue of being human and living on earth in a human society. "Experiential" should definitely NOT be taken in the empiricist sense as mere sense impressions that give form to the passive tabula rasa of the empiricists. We take experience as active functioning as part of a real world - as motivating what is meaningful in human thought (Lakoff 1988: 120).

Deuxièmement, le sens (meaning) implique une projection imaginative (et non une correspondance stricte de symboles abstraits avec des signes ou des objets), qui comprend la schématisation, la catégorisation, les conceptualisations métaphorique et métonymique et qui permet de passer de l'expérience corporelle structurée à l'élaboration de modèles abstraits.

La conceptualisation métaphorique nous intéresse tout particulièrement, car il est bien maintenant bien établi qu'elle constitue une composante essentielle de la théorisation scientifique, la métaphorisation vulgarisante ne constituant généralement qu'une extension de la conceptualisation métaphorique des scientifiques euxmêmes. Pour Lakoff, la conceptualisation métaphorique reflète directement la capacité du cerveau à fonctionner dans un environnement changeant, dont la description ne peut être prévue à l'avance. Elle correspond à un processus de pensée fondamental dans lequel la structure d'un cadre conceptuel source (source domain) se projette sur un cadre conceptuel cible (target domain), et qui se manifeste au plan linguistique dans des expressions métaphoriques ${ }^{3}$.

Nous mettrons en garde le lecteur quant au fait que la charge historique des travaux sur la métaphore induit immédiatement l'idée de déviance, ou de procédé rhétorique ou littéraire, ce qui n'est pas la façon d'envisager ici le phénomène métaphorique. Ceci est un problème récurrent. Nous ne saurions trop insister que là n'est pas le caractère définitoire de la métaphore conceptuelle, même si elle peut effectivement être utilisée à de telles fins. Par ailleurs, la distinction métaphore morte - métaphore vive faite par la présence ou l'absence de lexicalisation n'est pas un critère de définition ni de repérage de la conceptualisation métaphorique, c'est une propriété secondaire de l'expression linguistique métaphorique qui dépend de sa fréquence d'utilisation chez les locuteurs d'une langue. Enfin, si la conceptualisation métaphorique est sous-jacente à de nombreuses extensions de sens d'unités lexicales, elle ne saurait s'y réduire - d'une part parce que la focalisation de la réflexion est la conceptualisation et non l'unité lexicale, d'autre part parce qu'il existe d'autres formes de conceptualisations (métonymiques, par exemple). Lakoff a démontré, de façon convaincante, que la conceptualisation métaphorique est un processus cognitif généralisé 
qui passe souvent inaperçu, car elle s'exprime dans de nombreuses expressions de la vie de tous les jours, ce qui lui confère d'ailleurs sa puissante valeur de structuration cognitive.

\subsection{Talmy: factivité et fictivité}

Talmy (2000) développe notamment le modèle général de la fictivité, apportant l'idée fondamentale de «discrepancy within the cognition of a single individual»: deux représentations cognitives du même objet sont construites simultanément et sont perçues comme exclusives l'une de l'autre et formant une alternative, mais cette incompatibilité apparente est en elle-même source de sens. Sous l'angle linguistique, le modèle de la fictivité s'applique dans la mesure où l'une des représentations, factive, est plus véridique (elle correspond à la réalité perçue) et l'autre, fictive, est moins véridique (elle est évoquée par la forme linguistique). Un des exemples présentés par Talmy est celui du déplacement fictif, couplé à l'immobilité factive, évoqués par des expressions telles que: "The fence goes from the plateau to the valley». La forme verbale goes from évoque un déplacement, tandis que l'on «sait» par ailleurs que la clôture est immobile. Talmy soutient que c'est le fait de suivre un objet des yeux sur toute sa longueur qui autorise le recours à des expressions linguistiques évoquant un déplacement fictif. Ainsi que le souligne Talmy, ce modèle est parfaitement compatible avec les métaphores conceptuelles proposées par Lakoff et avec la gestalt, selon laquelle des perceptions apparemment incompatibles mènent à une expérience de dissonance et à des processus cognitifs qui tendent à la réduire. Nous verrons un peu plus loin que l'idée de dissonance est fondamentale dans le repérage des éléments linguistiques impliqués dans les modes de conceptualisation scientifique.

\subsection{Fauconnier: espaces mentaux et intégration conceptuelle}

Selon Victorri (2004), le cadre théorique développé par Fauconnier (voir par ex. Fauconnier et Turner 2002) est sans doute le plus général des grammaires cognitives, car il se situe dans une perspective centrée sur le discours, incluant la sémantique et la pragmatique. Deux concepts dominent les travaux de Fauconnier: les espaces mentaux et l'intégration conceptuelle. Un espace mental est une configuration cognitive construite par le sujet à partir des formes linguistiques, particulièrement dans un cadre discursif. L'intégration conceptuelle est le résultat de la fusion de deux configurations cognitives existantes: ainsi, c'est l'intégration des représentations fictive et factive qui permet au sujet de comprendre la phrase citée plus haut. Ce concept ajoute également un éclairage supplémentaire au traitement des modes de conceptualisation (métaphoriques comme métonymiques), puisque l'on pourrait dire que la construction du sens se réalise grâce à l'intégration des cadres conceptuels source et cible et des éléments des cadres ainsi mis en correspondance.

\section{Application à une approche cognitive de la traduction}

La cohérence de la sémantique cognitive avec ce qui semble être le plus pertinent dans les neurosciences actuelles nous paraît être un argument de poids en sa faveur. C'est pourquoi elle nous paraît constituer une source d'inspiration fondamentale pour la traductologie. En ce qui nous concerne, nous avons recours à la sémantique cognitive 
avec le projet de décrire des aspects fondamentaux de la traduction du discours spécialisé, en ciblant plus précisément la biomédecine, avec différents objectifs: (1) comme l'a déjà proposé Termmerman (2000), reconsidérer les postulats et les pratiques en matière de terminologie spécialisée; (2) accorder la prééminence à l'étude du sens à partir du discours dans ses dimensions référentielles, conceptualisantes et communicatives; (3) proposer des outils cognitifs pertinents au transfert linguistique, qui puissent améliorer l'appropriation du texte et du sens par le traducteur. Nous n'aborderons pas ici la question de la terminologie. Nous nous attarderons plutôt sur les deux derniers points.

\subsection{Le discours: référence, conceptualisation, communication}

Si le langage est étroitement lié à la cognition, et partant, la langue elle-même, étudier la langue «en elle-même et pour elle-même» selon le dogme issu de la Linguistique générale saussurienne, c'est-à-dire en la séparant de la pensée humaine, est discutable. Les langues ne constituent pas des systèmes indépendants de l'humain: les envisager ainsi constituent, au mieux, une «fiction opératoire», c'est-à-dire une objectivisation nécessaire pour légitimer leur étude selon une pensée cartésienne, séparant sans nuance possible le sujet de l'objet de son étude. Par ce postulat, tendrait-on à oublier que toute langue vivante n'existe que par sa connaissance partagée chez un certain nombre de locuteurs? Et que la norme ne vise (ou en tout cas elle le devrait) qu'à entériner la plus grande «intersubjectivité partagée», pour reprendre une expression utilisée par différents auteurs? En continuant avec les distinctions saussuriennes, peut-il exister une langue sans parole, sans discours?

L'histoire a de ces rebondissements inattendus: le principe de "la langue envisagée en elle-même et pour elle-même» serait en fait issu d'une déformation de la pensée de Saussure. Dans une ébauche très avancée intitulée De l'essence double du langage, mise au jour en 1996 et publiée en 2002 dans les Écrits de linguistique générale par Bouquet, le discours, le social et le subjectif sont étroitement liés à une «linguistique de la langue» (de Saussure 2002). L'époque est peut-être propice à une telle remise en question, et l'objectivisation transitoire des langues était sans doute une étape obligée de la légitimation des sciences du langage. Voilà qui vient apporter de l'eau au moulin de la traductologie, nécessairement focalisée sur le discours et dont la plus grande contribution aux sciences du langage a été précisément de souligner l'importance des facteurs culturels, sociaux, collectifs et individuels dans la production textuelle.

De façon générale, le discours se caractérise par trois dimensions fondamentales. La première concerne son contenu: de quoi parle-t-on? Il s'agit ici de se pencher sur la question de la référence et de la dénomination - autrement dit la terminologie. La deuxième concerne ce qui est dit du référent auquel renvoie le texte, et plus particulièrement la manière dont il est conceptualisé. La troisième dimension renvoie à la question: comment le dit-on? Il s'agit ici des composantes communicatives, que nous n'aborderons pas ici, bien que nous souscrivions à l'idée d'Eco selon laquelle «une référence ne peut être opérée que dans un processus de communication où un émetteur produit une expression pour un destinataire ou dans une situation spécifique» (1988/2006: 70). Ajoutons la problématique du transfert linguistique, qui devient la quatrième dimension percutant les trois autres. 
Lakoff n'est pas le seul à s'être opposé à l'objectif de formalisation mathématique de l'esprit, du langage et des langues. Eco suit le même chemin, bien qu'argumentant sous l'angle de la sémiotique et non à partir de postulats psychologiques ou neurobiologiques: il nous invite à voir «le texte comme un acte de référence» à un «monde possible (le monde de notre expérience étant l'un des nombreux mondes possibles» (Eco 1988/2006: 64-76). Il nous paraît justifié de compter parmi ces mondes possibles les mondes modélisés issus de la théorisation scientifique, puisqu'ils ont pour objet d'organiser de façon intelligible un ensemble de données du monde expérientiel, que ces données soient acquises de façon directe ou indirecte par le biais de l'expérimentation. En réponse au constructivisme extrême, le problème de fond n'est pas la nonréalité du monde, c'est celui de la variabilité des perceptions interindividuelles, de leur caractère partiel, ce qui amène une multitude de points de vue différents.

L'objectif d'organisation intelligible réclame un effort de conceptualisation de l'objet scientifique: les théories se combattent en fin de compte sur les différents modes de conceptualisation autorisés par les observations. Par conséquent, le discours scientifique présente la particularité de traiter d'un objet dont la conceptualisation est en constante évolution. Ce point est fondamental: le caractère référentiel du discours s'accompagne d'une dimension imaginative (au sens de Lakoff) dans lequel n'en déplaise aux objectivistes, qui seront déçus - les procédés de pensée tels que conceptualisations métaphorique et métonymique sont fondamentaux. Cet aspect du discours scientifique est maintenant bien établi et confirmé par de nombreux travaux, dont les nôtres dans le domaine biomédical.

Pour Eco, la métaphore ne peut opérer qu'en discours: on ne peut percevoir la métaphore que si l'on «entend le texte comme un acte de référence et le compare à un état de fait» (Eco 1988/2006: 76). Ce n'est que par la prise en compte de la référence dans le monde possible des référents anatomiques que le caractère de véridicité moindre, "fictif», d'une expression telle que l'artère court le long du muscle peut être perçue, si l'on admet que courir, unité polysémique, renvoie en premier lieu à une situation dans laquelle une entité animée se déplace à une certaine vitesse. Nous reviendrons ce point un peu plus loin.

\subsection{Les modes de conceptualisation}

La question des modes de conceptualisation nous paraît centrale dans un contexte de traduction en général, et spécialisée en particulier. Saisir la conceptualisation en jeu dans un texte scientifique est une étape clé de la compréhension. Nous nous rattachons, pour l'étude des modes de conceptualisation métaphorique, aux travaux de Lakoff dans sa composante de projection de cadre conceptuel source sur un cadre conceptuel cible. (Il est clair toutefois que la conceptualisation métonymique est également très importante et méritera qu'on s'y attarde ultérieurement.) La sémantique cognitive ne fournit cependant pas d'emblée de méthode d'analyse systématique partant des formes linguistiques et du texte. Nous avons par conséquent cherché à établir une méthode permettant de cerner les conceptualisations parcourant la biologie cellulaire (Vandaele et Lubin 2005; Vandaele et coll. 2006) et l'anatomie (Lubin 2006) et avons ainsi été amenée à définir le concept d'indice de conceptualisation. La méthode a été décrite en détail ailleurs (Vandaele et Boudreau 2006): en résumé, le travail se réalise à l'aide d'une technique d'annotation sémantique de corpus en 
format XML, autorisant ultérieurement tant une analyse qualitative que quantitative des données recueillies. Le repérage des indices de conceptualisation par le sujet annotateur constitue le point clé de la méthode.

\subsubsection{Les indices de conceptualisation: factivité et fictivité}

Le repérage des indices de conceptualisation repose sur la perception d'un effet de «dissonance cognitive» (Vandaele et Lubin 2005; Vandaele et coll. 2006). Cette idée est très voisine du concept de fictivité proposé par Talmy: dans la phrase l'artère court le long du muscle, le verbe courir évoque l'idée d'un déplacement, tandis que nous savons par ailleurs que l'artère reste immobile (au besoin en consultant des ouvrages ad hoc si notre connaissance de l'anatomie est rudimentaire). Nous avons appelé indice de conceptualisation la forme linguistique induisant la coexistence d'au moins deux représentations, l'une factive et l'autre fictive. Une façon de traiter cette question est de dire que courir, dans ce contexte, a subi une extension de son sens premier, et qu'une nouvelle acception, métaphorique, existe. Pour Talmy, la représentation factive correspond au sens per se de l'unité lexicale (courir dans notre exemple). Il nous paraît préférable d'envisager que la forme linguistique a plusieurs acceptions, l'une correspondant à la situation de déplacement, l'autre à la situation d'immobilité décrite au moyen d'un déplacement fictif, et que ces deux acceptions sont mobilisées simultanément, mais avec des valeurs différentes. Distinguer les différentes acceptions des formes linguistiques est puissant, car cela autorise la description de leurs propriétés respectives (traits sémantiques, structure actancielle, régime syntaxique, collocations ${ }^{4}$ ) et éventuellement leur consignation rigoureuse dans un dictionnaire. Ce qu'il faut retenir ici, c'est l'idée que l'indice de conceptualisation est repéré par un locuteur donné lorsque ce dernier perçoit que «quelque chose est conceptualisé comme autre chose».

Cette approche méthodologique irritera sans aucun doute les tenants des critères linguistiques «objectifs", mais l'objectivisme en matière linguistique nous paraît suffisamment réfuté pour qu'il soit préférable de se donner les moyens de traiter la subjectivité apparente qui lui est liée. De fait, Talmy souligne que la perception de la fictivité est très variable d'un individu à l'autre, et nous ajouterons qu'elle sera sans doute plus fiable chez un locuteur natif. Le traducteur ajoutera donc à ses compétences celle de pouvoir repérer les indices de conceptualisation dans la langue source et de les manier dans la langue cible. En recherche, la façon idéale de traiter rigoureusement le problème de repérage des indices de conceptualisation consistera à vérifier la constance du repérage des indices de conceptualisation chez un groupe statistiquement représentatif des locuteurs natifs d'une langue. C'est non seulement la solution classique à un problème envisagé sous l'angle de l'induction, mais également celle qui rend compte de l'intersubjectivité partagée du phénomène (les psychologues sont habitués à gérer ce genre de problèmes; voir par ex. Le Ny, 1979; 2005).

Nous avons déterminé deux grandes catégories d'indices de conceptualisation: les dénominations conceptualisantes et les IC phraséologiques.

\subsubsection{Les dénominations conceptualisantes}

Selon Kleiber (1996: 574-575), la « relation de dénomination appartient au domaine des relations signes $\longleftrightarrow \longrightarrow$ choses et se place ainsi du côté des relations référentielles: référer à, renvoyer à, désigner, représenter, dénoter, etc. Elle se sépare toutefois de ces 
relations de désignation par la nécessité d'un acte de dénomination préalable entre le signe et la chose dénommée. Pour qu'une relation relations signe $\longleftrightarrow \longrightarrow$ chose soit dénominative, il faut en effet au préalable qu'un lien référentiel particulier ait été instauré entre la chose $x$, quelle qu'elle soit, et le signe $\mathrm{X}$, alors qu'une telle exigence n'est nullement requise par la relation de désignation ». Kleiber souligne que cette association est faite pour durer. Or, lorsque ce qui préside à la dénomination est un acte de conceptualisation métaphorique, l'association peut effectivement durer, mais la motivation se perd progressivement. Ainsi, lorsque Robert Hooke observa pour la première fois la structure du liège aux débuts de la microscopie optique en 1655, il observa de petites cavités vides, qui lui rappelèrent les cellules monacales. À l'époque, on était à la recherche des unités de base des tissus vivants: Hooke nomma ces cavités, qu'il prit - à tort - pour les traces de ces unités, cell. Il s'agissait d'un acte de dénomination conférant à cell le statut d'indice de conceptualisation métaphorique. Avec le temps, le référent associé à la dénomination prend le dessus sur la motivation qui en est à l'origine. C'est pourquoi, en synchronie, le repérage de néologismes indices de conceptualisation de type dénominatif a une probabilité plus élevée de succès qu'en diachronie. La forme linguistique devient polysémique: si elle persiste dans l'usage, le lien entre le sens premier et le nouveau sens risquera de se perdre. Ces formes linguistiques, ultimement, ne seront plus perçues comme des indices de conceptualisation de façon courante, seule une analyse étymologique poussée permettrait d'en retrouver éventuellement la trace. Dans le cas des dénominations conceptualisantes, la métaphore est directe, car elle opère sur le référent ainsi nommé et elle est indépendante du discours.

\subsubsection{Indices de conceptualisation phraséologiques}

Nous classons dans cette catégorie les indices de conceptualisation prédicatifs qui, dans un discours donné, induisent une conceptualisation métaphorique des référents dont le nom est en position actancielle (Vandaele 2004; Vandaele et Lubin 2005; Vandaele et coll. 2006). L'exemple présenté plus haut est représentatif de cette catégorie: «l'artère court le long du muscle». Le référent |artère| dénoté par le terme artère est conceptualisé comme une entité se déplaçant de façon fictive. Il est intéressant de remarquer que de nombreux verbes évoquant un déplacement fictif sont utilisés pour décrire les artères et les veines, mais aussi les cours d'eau, ce qui complexifie les conceptualisations métaphoriques: il est en effet courant de conceptualiser les vaisseaux sanguins comme un réseau fluvial, et cela se manifeste dans l'expression langagière. Ce type d'indices de conceptualisation est fondamental pour la phraséologie et l'idiomaticité des textes. Beaucoup de travail reste à faire pour caractériser, à l'aide d'indices de conceptualisation, le transfert linguistique de l'idiomaticité. Enfin, dans ce cas, la conceptualisation métaphorique est indirecte, car elle opère sur les actants de l'indice de conceptualisation et non sur celui-ci, et elle a besoin du discours pour se révéler.

3.2.3. Réseaux lexicaux, modes de conceptualisation et intégration conceptuelle

Les indices de conceptualisation forment des réseaux qui rendent cohérent un mode de conceptualisation donné dans un texte ou un domaine spécialisé. Plus le réseau est riche, plus la conceptualisation est prégnante. Nous avons montré qu'en biologie 
cellulaire des réseaux lexicaux riches et diversifiés témoignaient de la conceptualisation des cellules et des molécules comme des personnes, mais que les indices de conceptualisation constituant chacun des deux réseaux différaient, en anglais comme en français ${ }^{5}$ (Vandaele et coll. 2006). Ainsi, les conceptualisations, dans leurs spécificités, seront différentes. En anatomie, les réseaux d'indices de conceptualisation verbaux utilisés pour décrire le positionnement des nerfs, des artères, des veines et des muscles présentent également des différences, dont certaines sont liées à la fonction des entités en cause: par exemple, le verbe irriguer est cohérent avec la conceptualisation des artères comme des cours d'eau, mais il ne s'utilise pas pour les veines. À l'inverse, drainer est spécifique aux veines, qui sont elles aussi conceptualisées comme des cours d'eau (Lubin 2006). Le fait que les artères amènent le sang vers la périphérie et que les veines le ramènent au cœur explique cet usage spécifique des verbes.

D'autres modes de conceptualisation peuvent être mis en évidence. On trouve par exemple, en biologie cellulaire:

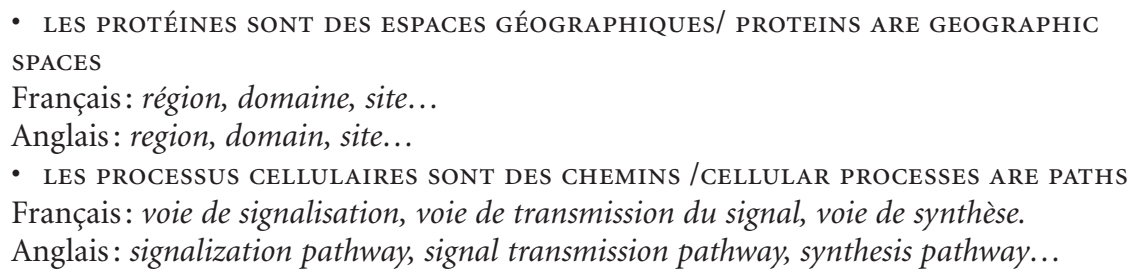

Enfin, il est remarquable que le discours témoigne de l'expression simultanée de plusieurs conceptualisations métaphoriques, ce qui correspond à une intégration conceptuelle caractéristique d'un domaine de spécialité:

«Les membres de la famille de Bcl-2 et les protéases à activité cystéine nommées caspases sont des effecteurs principaux de la machinerie apoptotique, présente dans toutes les cellules.»

"They physically transfer the signal from the point at which it is received to the cell machinery that will make the response.»

Dans ces deux exemples, on relève la conceptualisation LES MOLÉCULES SONT DES PERSONNes (membres de la famille, activité), LES CELLULES SONT DES USINES/CELLS ARE FACTORIEs (machinerie, machinery), CELLS ARE COMMUNiCATION SySTEMs (signal).

On remarque de plus que les modes de conceptualisation Les CELLULES SONT DES usines et CELls ARE FaCtORIES sont compatibles, mais ce pourrait ne pas être le cas. Il est tout à fait possible d'envisager des situations où un référent est conceptualisé de façon différente dans deux cultures données - ce que la stylistique comparée a souvent catégorisé comme «un découpage de la réalité différent». Le travail sur le transfert linguistique devrait donc s'envisager à partir d'un travail global sur les modes de conceptualisation et les réseaux lexicaux qui leur sont associés.

\section{Remises en question et perspectives en traductologie}

Les résultats obtenus nous paraissent susciter quelques remises en question.

- Si certaines unités sont effectivement caractéristiques d'un domaine de spécialité (les termes renvoyant aux notions du domaine), la mise en évidence des réseaux lexicaux constitués par les indices de conceptualisation montre que l'ensemble du lexique est 
en fait potentiellement mobilisable en discours. Les unités lexicales concernées n'acquièrent pas nécessairement un sens nouveau (bien que cela puisse être le cas), mais elles n'en sont pas moins essentielles, tant pour la compréhension du texte que pour la décision traductionnelle. Caractériser un domaine par des unités linguistiques qui lui seraient spécifiques ne paraît pas mener à une description complètement pertinente pour un contexte de traduction, bien que cela puisse se justifier dans une optique de construction de dictionnaire ou de base de données terminologiques.

- Dans cette optique, l'opposition langue générale et langue de spécialité ne reflète pas véritablement la réalité, sauf si l'on admet une intersection notable entre les deux. (On constate d'ailleurs que les corpus dits de «langue générale» sont constitués d'un ensemble de sous-corpus appartenant à des domaines différents.) La caractérisation d'un domaine de spécialité se fait au niveau conceptuel, par une nouvelle combinatoire de modes de conceptualisation appliqués aux référents spécifiques au domaine.

- Les indices de conceptualisation phraséologiques sont essentiels pour conférer l'idiomaticité au texte. Approfondir le moyen de repérer et d'utiliser les indices de conceptualisation dans les langues source et cible constitue un objectif pour la constitution de ressources lexicographiques et terminologiques.

- La perspective traductionnelle soulève la question des modes de conceptualisation véhiculés dans différentes langues. En biologie cellulaire, l'anglais et le français présentent une grande compatibilité. Mais plus les domaines seront marqués culturellement, plus les modes de conceptualisation vont varier. La mise en évidence des réseaux lexicaux sous-jacents à un mode de conceptualisation montre qu'il faut prendre en compte toutes ses composantes. La question de l'équivalence devra donc être revisitée à la lumière de l'approche cognitive.

- Enfin, pour terminer, une hypothèse à vérifier: la différence entre traducteurs confirmés et traducteurs débutants pourrait-elle se ramener, essentiellement, à la connaissance des modes de conceptualisation d'un domaine, et de la phraséologie qui l'accompagne?

Bien que les questions de processus de traduction, de sens et de représentations conceptuelles aient déjà suscité un intérêt marqué chez différents chercheurs en traductologie, ce n'est que relativement récemment que les recherches en traduction semblent s'ouvrir plus largement à une réflexion fondée sur une approche cognitive. La plupart du temps, ces études se penchent sur les processus à l'œuvre, les nouvelles techniques d'imagerie venant compléter les études déjà réalisées à l'aide des protocoles de verbalisation (voir les autres auteurs du présent numéro; Danks et coll., 1997; Lee-Jahnke, 2006), mais nous croyons que les études à base de corpus fondées sur une approche cognitive peuvent également renouveler la recherche et la pratique (Rydning 2002, 2005). Il s'agit d'un courant d'exploration novateur en traduction spécialisée, d'autant que cette dernière a été quelque peu le parent pauvre de la traductologie, peut-être en raison du développement des autres courants de la discipline, de l'importance prise par la terminologie (ce qui a pu occulter d'autres aspects de la traduction spécialisée) et du fossé séparant le monde universitaire de la pratique professionnelle.

Selon nous, la traduction humaine est avant tout une activité cognitive, en ce qu'elle mobilise les ressources de l'intelligence humaine dans nombre de ses dimensions. La traduction spécialisée, du fait qu'elle concerne le plus souvent des domaines de connaissances difficiles d'accès, présente de nombreuses particularités, notamment au plan de l'apprentissage et des méthodes de travail. À ce titre, elle constitue un terrain propice non seulement à l'examen du transfert linguistique, mais aussi à l'étude des processus de réflexion et de raisonnement sur les textes, la langue et les 
connaissances - biomédicales en ce qui nous concerne. Par conséquent, elle se doit de participer au courant actuel des linguistiques cognitives et des travaux de sémantique, tant pour en bénéficier que pour apporter sa propre pierre à l'édifice et mettre à l'épreuve de la réfutation les différentes approches proposées. Il importe donc de mettre en œuvre un programme ouvrant de nouvelles voies de recherche et menant à l'élaboration de nouveaux outils de réflexion sur la langue et le transfert linguistique spécialisé. De plus, ces outils doivent certes être suffisamment puissants pour alimenter la réflexion théorique et l'expérimentation, mais il nous paraît essentiel, en cette ère où le traducteur doit de plus en plus se resituer face aux techniques de traitement automatique de la langue, qu'ils puissent être transférables à l'enseignement et à la pratique professionnelle, c'est-à-dire faire partie de la «boîte à outils » cognitifs du praticien.

Pour terminer, revenons à la philosophie. En quoi Spinoza est-il particulièrement intéressant dans un cadre traductologique? Descartes, dans son opposition entre le divin et la réalité des choses connues, crée une transcendance absolue et totalisante: «[...] l'intelligible trouvant sa source dans l'inintelligibilité. Le divin est le nom donné aux conditions radicales de toute intelligibilité. Ainsi, la compréhension, dans sa quête du divin (totalisation), requiert-elle la transcendance [...]. C’est précisément à la théorie cartésienne de l'erreur comme mauvaise liaison entre notre volonté infinie, à laquelle il incombe de chercher puis, surtout, de juger, et notre entendement fini, qui conçoit les idées vraies, mais ne peut les affirmer, que Spinoza s'en prend avec le plus de violence» (Rizk 2006: 6-9).

Si la vérité n'est accessible que par l'accès à la transcendance, elle est toujours ailleurs. Elle interdit, sous couvert d'objectivité, toute prise de décision personnelle. Or, l'opération traduisante chez l'humain est une opération cognitive de haut niveau, de caractère "privé», à travers laquelle on peut appréhender la variabilité interindividuelle des processus mentaux. À cause de cette variabilité, elle n'est pas crédible dans la perspective objectiviste. Et l'injonction de douter, trop souvent véhiculée dans l'enseignement de la traduction et intériorisée de façon si puissante qu'elle tétanise l'intelligence, est le pire héritage cartésien qui puisse être transmis aux traducteurs en devenir. Mais trop souvent on remplace l'injonction du doute par celle de l'intuition, ce qui mène malheureusement à entretenir une croyance en une forme de connaissance immédiate, finalement tout aussi transcendante, tout aussi déconnectée des processus cognitifs, et qui laisse l'apprenti-traducteur complètement décontenancé. L'intuition n'est rien d'autre que le résultat intégré des processus cognitifs sous-tendant l'intelligence, et l'on aura tout intérêt, si on ne veut pas rester pris dans la tentation totalisante de la transcendance ou de l'intuition, à mieux connaître la diversité de ces processus. Dans ce contexte, le raisonnement logique garde sa place (ce n'est pas parce que le cerveau n'est pas un ordinateur qu'il faut abandonner la cohérence logique de certains processus cognitifs - les niveaux en jeu sont totalement distincts), mais il faut prendre en compte les autres formes de la connaissance, dont la projection imaginative. Le repérage des indices de conceptualisation et la compréhension de la conceptualisation métaphorique, des principes de factivité et de fictivité, ainsi que de l'intégration conceptuelle sont quelques armes de plus pour réfléchir au transfert linguistique.

Spinoza, écartant d'emblée superstitions et illusions, se rattache, pour le meilleur et pour le pire, à la réalité et à l'expérience: «La tension de l'expérience nous délivre paradoxalement des illusions comme des arrière-mondes. C'est, en effet, l'expérience 
qui porte au jour l'étonnante opportunité d'une politique de la liberté, en fonction d'une puissance que les individus peuvent s'approprier et exprimer comme la nécessité adéquate, comprise en sa vérité ontologique, de leur activité» (Rizk 2006: 179). Expérience (des mondes possibles) et appropriation (du sens), voilà les maîtres mots qui devraient guider toute pratique de la traduction.

\section{NOTES}

* Nous remercions le Conseil de recherches en sciences humaines du Canada et le Fonds québécois de la recherche sur la société et la culture, ainsi que l'Université ionienne. Nous remercions également Sylvie Boudreau, Leslie Lubin, Mariana Raffo et Elizabeth Marshman pour leur contribution aux travaux de recherche.

1. «Selon ce point de vue [la philosophie idéaliste], le monde n'est perçu qu’à travers l'esprit, et il se peut donc que la matière n'existe pas - que seul l'esprit existe, comme l'a suggéré Berkeley. Ayant entendu cela, le Dr Johnson donna un coup de pied dans une pierre et déclara: «Je le réfute ainsi!»» (Edelman 1992: 327).

2. Dans l'optique de Damasio, l'émotion et le sentiment, confondus de façon courante, sont distincts: l'émotion doit être comprise comme étant la manifestation corporelle (publique, donc) d'un état particulier, tandis que le sentiment se manifeste au plan de l'esprit, et reste ainsi fondamentalement privé (Damasio 2003: 34).

3. Pour ne pas alourdir inutilement le texte, nous renvoyons le lecteur aux travaux de Lakoff, tout particulièrement son article de 1993, qui explicite clairement la distinction entre la vision classique et la vision de la sémantique cognitive: "The word "metaphor" has come [...] to mean a "cross-domain mapping in the conceptual system". The term "metaphorical expression" refers to a linguistic expression (a word, a phrase, or sentence) that is the surface realization of such cross-domain mapping (this is what the word "metaphor" referred to in the old theory).»

4. Nous avons utilisé pour cela les principes de la Lexicologie explicative et combinatoire de la Thérie Sens-Texte de I. Mel' uk qui, fondée sur des principes de logique formelle, est particulièrement puissante pour décrire les unités lexicales prédicatives. Nous nous sommes déjà expliquée sur le recours à des outils issus de la logique (Vandaele et coll. 2006) et suivons en cela Le Ny, qui se réfère plutôt au formalisme de Davidson dans un but similaire (Le Ny 1979; 2005).

5. LES CELLULES SONT DES PERSONNES / CELLS ARE PERSONS

Français: communauté, coloniser, mort/mourir, suicide, parenté, partenaire, famille, population, coopérer, donner naissance, responsable, rôle...

Anglais: community, migration, death/to die, suicide, decision, family, population...

LES MOLÉCULES SONT DES PERSONNES / MOLECULES ARE PERSONS

Français: agir, reconnaître, coopérer, recruter, s'exprimer, actif, inactif, rôle, responsable...

Anglais: to act as, to recognize, to recruit, active, inactive, responsible...

\section{RÉFÉRENCES}

Anscombre, J.-C. (1998): «Regards sur la sémantique française contemporaine. In Diversité de la (des) science(s) du langage aujourd'hui», Langages 129, p. 37-51.

Atlan, H. (2002): La science est-elle humaine?, Paris, Bayard.

Changeux, J.-P. et P. Ricceur (1998): Ce qui nous fait penser, La Nature et la Règle, Paris, Odile Jacob.

Damasio, A. R. (2003): Looking for Spinoza: Joy, Sorrow, and the Feeling Brain, Orlando, Harcourt (traduction française par J.-L. FIdEL (2005): Spinoza avait raison. Joie et tristesse, le cerveau des émotions, Poches Odile Jacob).

Danks, J., Shreve, G., Fountain S. and M. McBeath (eds.) (1997): Cognitive Processing in Translation and Interpreting. Applied Psychology, vol. 3, Thousand Oaks and London, Sage Publications.

Descartes, R. (1637): Discours de la méthode, Paris, Classiques Larousse (réédition de 1994, commenté par A. RoBinet, Larousse).

Edelman, G. (1992): Bright Air, Brilliant Fire: On the Matter of Mind, New York, Basic Books (traduction française par A. Gershenfeld (1992): Biologie de la conscience, Paris, Odile Jacob). 
Edelman, G. (2004): Wider than the sky - The phenomenal gift of consciousness, New Haven/ London, Yale University Press.

de Saussure, F. (2002): Écrits de linguistique générale, Bouquet, S. et R. Engler (dir.), Paris, Gallimard.

Eco, U. (1988/2006): Sémiotique et philosophie du langage, Paris, PUF.

Fauconnier, G. et M. Turner (2002): The Way We Think - Conceptual Blending and the Mind's Hidden Complexities, New York, Basic Books.

Fodor, J. (1983): The modularity of Mind, Cambridge, MIT Press (traduction française par A. Gershenfeld (1986): La modularité de l'esprit, Paris, Éditions de Minuit).

Fodor, J. (2000): The Mind Doesn't Work That Way, MIT Press, Cambridge (traduction française par C. Tiercelin (2003): L'esprit, ça ne marche pas comme ça, Paris, Éditions Odile Jacob).

Fuchs, C. (2004): «Pour introduire la linguistique cognitive», in Fuchs, C. (dir.) La linguistique cognitive, Paris, Éditions Ophrys, Éditions de la Maison des sciences de l'homme.

JaQuet, C. (2004): L'unité du corps et de l'esprit. Affects, actions et passions chez Spinoza, Paris, PUF.

Kleiber, G. (1996): «Noms propres et noms communs: un problème de dénomination », Meta 41-4, p. 567-589.

LAKofF, G. (1977): «Linguistic Gestalts», in BEACH, W.A. et al (eds.), Papers from the 13th Regional Meeting, Chicago Linguistic Society, April 14-16, 1977, Chicago Linguistic Society, University of Chicago, Chicago, p. 236-287.

Lakoff, G. (1988): "Cognitive Semantics», in Eco, U., Santambrogio, M. and P. Violi (eds), Meaning and Mental Representations, Bloomington/Indianapolis, Indiana University Press.

Lakoff, G. (1993): «The Contemporary Theory of Metaphor», in Ortony, A. (ed.), Metaphor and Thought, Cambridge University Press.

Lakoff, G. et Johnson, M. (1999): Philosophy in the Flesh - The Embodied Mind and its Challenge to Western Thought, New York, Basic Books.

Lee-JAhnke, H. (dir.) (2005) : «Processus et cheminements en traduction et interprétation», Meta $50-2$.

Le Ny, J.-F. (1979): La sémantique psychologique, Paris, Presses Universitaires de France.

Le Ny, J.-F. (2005): Comment l'esprit produit du sens, Paris, Odile Jacob.

Lubin, L. (2006): Étude des métaphores conceptuelles utilisées dans la description des structures anatomiques, mémoire de maîtrise, Université de Montréal.

Rizk, H. (2006): Comprendre Spinoza, Paris, Armand Colin.

Rouveret, A. (2004): «Grammaire formelle et cognition linguistique», in Fuchs, C. (dir.) La linguistique cognitive, Paris, Éditions Ophrys, Éditions de la Maison des sciences de l'homme.

Rydning, A.F. (2002) : «Concept métaphorique et expression métaphorique dans une perspective cognitiviste», Romansk Forum 16-2, p. 723-733.

Rydning, A. F. (2005): «The Return of Sense on the Scene of Translation Studies in the Light of the Cognitive Blending Theory", Meta 50-2, p. 393-404.

SpinozA, B. (1659-1677): Euvres (traductions et notes par C. Appunn), Paris, Garnier.

Talmy, L. (2001): «Fictive Motion in Language and "Ception"», Toward a Cognitive Semantics 1, p. 99-175.

Temmerman, R. (2000): Towards New Ways of Terminology Description: The Sociocognitive Approach, Amsterdam/Philadelphia, John Benjamins.

VArela, F. J. (1989): Connaître les sciences cognitives. Tendances et perspectives, Paris, Éditions du Seuil.

Vandaele, S. (2004): «Deciphering Metaphorical Conceptualization in Biomedicine: Towards a Systematic Analysis", in Rogers, M. et K. Ahmad (eds) New Directions in LSP studies, Proceedings of the 14th European Symposium on Language for Special Purposes, Surrey, 18-22 août 2003, Communication, Culture, Knowledge, p. 195-202.

VANDAELE, S. et L. Lubin (2005): «Approche cognitive de la traduction dans les langues de spécialité: vers une systématisation de la description de la conceptualisation métaphorique» Meta 50-2, p. 415-431. 
Vandaele, S., Boudreau, S., Lubin, L. et E. Marshman (2006): «La conceptualisation métaphorique en biomédecine: indices de conceptualisation et réseaux lexicaux», Glottopol 8, $<$ http://www.univ-rouen.fr/dyalang/glottopol/numero_8.html $>$.

Vandewelle, B., Busschaert B. et B. Meurin (2003): Des esprits animaux aux neurotransmetteurs, qui sait ce que peut le corps?, XXXII Journées Annuelles de Thérapie Psychomotrice, Colloque Corps et culture, Lille, 2 octobre 2003.

Victorri, B. (2004) : Les grammaires cognitives. In: Fuchs, C. (dir.) La linguistique cognitive, Cogniprisme, Éditions Ophrys, Éditions de la Maison des sciences de l'homme, Paris, p. 73-98. 